\title{
Increased serum soluble leptin receptor levels in children and adolescents with type 1 diabetes mellitus
}

\author{
J Kratzsch, A Deimel ${ }^{1}$, A Galler ${ }^{1}$, T Kapellen ${ }^{1}$, A Klinghammer ${ }^{2}$ and W Kiess ${ }^{1}$ \\ Institute of Laboratory Medicine, Clinical Chemistry and Molecular Diagnostics, University of Leipzig, Leipzig, Germany, ${ }^{1}$ Hospital for Children and \\ Adolescents, University of Leipzig, Leipzig, Germany and ${ }^{2}$ Children's Hospital, Chemnitz, Germany
}

(Correspondence should be addressed to J Kratzsch, Institute of Laboratory Medicine, Clinical Chemistry and Molecular Diagnostics, University Hospital, Paul-List-Str.13-15,D-04103 Leipzig, Germany; Email: kraj@medizin.uni-leipzig.de)

\begin{abstract}
Objective: We investigated whether or not serum levels of the soluble leptin receptor (sOB-R) and leptin are related to anthropometric and metabolic changes during pubertal development of children and adolescents with type 1 diabetes mellitus.

Design and methods: Blood levels of sOB-R, leptin and HbA1C, as well as body-mass index (BMI), diabetes duration and daily insulin doses, were determined in 212 (97 girls; 115 boys) children with type 1 diabetes mellitus and compared with the sOB-R serum levels in 526 healthy children and adolescents.

Results: OB-R serum levels and parallel values of the molar ratio between sOB-R and leptin were significantly higher in children with diabetes than in normal children $(P<0.05)$ in almost all investigated Tanner stages. Furthermore, in the entire group of patients, we demonstrated statistically significant correlations $(P<0.02)$ between sOB-R and the duration of diabetes $(r=0.30)$, HbAlc levels $(r=0.32)$ and the insulin dose $(r=0.18)$. Multiple-regression analysis revealed that HbA1c (12.4\%), height $(7.9 \%)$ and duration of diabetes $(8.7 \%)$ contributed to $29 \%$ variance of sOB-R in diabetic children.

Conclusions: Our data suggest that poor glycemic control in diabetes may lead to increased serum levels of sOB-R. This regulation of sOB-R appears to be independent of leptin, but may have an impact on leptin action. The consequently developing molar excess of sOB-R related to leptin could reduce leptin sensitivity and may, therefore, influence leptin-related anthropometric and metabolic abnormalities.
\end{abstract}

European Journal of Endocrinology $151475-481$

\section{Introduction}

The $o b$ gene product, leptin (1), is mainly secreted by the adipose tissue and acts predominantly by binding to hypothalamic cells. The biologic effects of leptin include suppression of food intake and increase of energy expenditure, and, consequently, the regulation of body weight $(2-5)$. Several studies have suggested that leptin also influences sexual progression through puberty (6-8). Leptin serum levels are related to body fat mass, body-mass index (BMI), gender and pubertal stage. In both sexes, leptin serum concentrations are increasing before the onset of puberty. In girls, leptin rises steadily until the end of sexual maturation. In boys, leptin levels decline in puberty after an initial rise later. This particular pattern can be partially explained by the effect of steroid hormones such as testosterone upon leptin expression $(6,9-12)$. Impaired growth and pubertal delay have been observed in children with diabetes (13-15). These children are also more inclined to develop obesity, even if they are treated with modern insulin therapy. The mechanism of weight gain in subjects with type 1 diabetes mellitus (DM) has not yet been examined; however, there are suggestions of an association between weight gain and hyperinsulinemia, which is reflected by correlations between the insulin dose and the frequency of insulin injections (16). Moreover, a recent study revealed that increased levels of leptin, indicating leptin resistance, may explain weight gain, especially in girls with diabetes (17). However, the leptin data of patients with diabetes compared with healthy controls are controversial during pubertal development. There is no unique picture of increased serum leptin levels (18-22). For better understanding of the correlation between the leptin axis and anthropometric as well as metabolic abnormalities of children with diabetes, we assessed the role of the soluble leptin receptor (sOB-R) as a potential modulator of leptin actions. The function of sOB-R has not been fully elucidated so far $(23,24)$. Like other hormone binding proteins of the cytokine family, sOB-R could prolong the half-life and, therefore, the bioavailability of its ligand 
leptin in blood. However, as a molar excess of sOB-R over leptin suppressed leptin bioactivity in vitro, the interaction between sOB-R and leptin may be a causal factor in the pathogenesis of weight gain in vivo (25). For further examination of this issue, we measured serum sOB-R and leptin levels in a large cohort of children and adolescents with diabetes, and we compared these data with sOB-R and leptin levels of healthy controls.

\section{Subjects and methods}

\section{Patients and controls}

A total of 212 patients (97 girls and 115 boys, age range: $2.8-19.3$ years; median: 12.7 years), diagnosed with type $1 \mathrm{DM}$ by the criteria of the American Diabetes Association (ADA) (26), were included in our study (Table 1). Eighty-two were patients at the Hospital for Children and Adolescents, University of Leipzig, Leipzig, Germany, and 130 patients came from the Children's Hospital of Chemnitz, Germany. All patients had diabetes duration of more than 5 months and were receiving exogenous insulin, administered by 3-5 subcutaneous injections daily. They demonstrated only in very rare cases $(<1 \%)$ clinical symptoms or signs of neuropathy, nephropathy, retinopathy or severe metabolic decompensation such as ketoacidosis. None of them had additional chronic or acute illness. Apart from insulin, no other permanent medication was used. None of the patients paid special attention to diet apart from the usual diabetes-related nutrition recommendations for a carbohydrate-balanced, lipidpoor and normal-protein diet. Reference values from children with comparable age and pubertal stage were taken from a previous study published by our group $(n=526)$ (24). All patients or their parents gave written, informed consent to participate in the study. The University of Leipzig ethics committee approved this study.

\section{Methods}

Clinical examination In all patients, clinical and anthropometric data were recorded on a structured data-sheet during routine examination. Weight was measured with light underwear to the nearest $0.1 \mathrm{~kg}$ on a calibrated balance-beam scale. Height was measured to the nearest $0.1 \mathrm{~cm}$ with a standardized measuring device (Keller system 2). BMI was calculated as body weight $(\mathrm{kg})$ divided by height $(\mathrm{m})$ squared. BMI s.D. Scores (SDS) levels were calculated according to the German Reference Data of Kromeyer-Hausschild from 2002 (27). Pubertal development was assessed according to Tanner (28). The whole set of data is demonstrated in Table 1.

Biochemical analysis Blood samples for leptin, sOB-R and $\mathrm{HbA} 1 \mathrm{C}$ were collected from a cubital vein at 0800-1200h. Samples were centrifuged at $4000 \mathrm{U}$ for $20 \mathrm{~min}$ and stored at $-25^{\circ} \mathrm{C}$ until analysis. $\mathrm{HbA1c}$ was measured by the Tina-quant technique (Roche Diagnostics Mannheim, Germany).

\section{Serum parameter of the leptin axis}

Leptin was measured by a specific 'in-house' radioimmunoassay (RIA) (29). The sensitivity of the RIA was $0.2 \mathrm{ng} / \mathrm{ml}$ (2 S.D. of the leptin-free standard matrix, $n=12$ ). The RIA proved to be precise, as its intra-assay and interassay coefficients of variation were both below $12.5 \%$ in the range $1-8 \mathrm{ng} / \mathrm{ml}$ leptin.

sOB-R was measured with a newly developed inhouse ligand-immunofunctional assay (LIFA) (24). The lowest detectable sOb-R concentration in the assay was calculated to be less than $2 \mathrm{ng}$ per ml. The intra-assay and interassay coefficients of variation for two control samples were lower than $11.7 \%(n=10)$. The molar sOB-R/leptin ratio was calculated by the measured levels of sOB-R divided by the leptin values and multiplied by 0.123 , the ratio between the molecular weights of both parameters (25).

Table 1 Anthropometric, biochemical and clinical data characterizing the patients with type 1 diabetes mellitus. Data were expressed as mean \pm S.D. or median and quartiles (BMI).

\begin{tabular}{|c|c|c|c|c|c|c|c|}
\hline $\begin{array}{l}\text { Tanner } \\
\text { stage }\end{array}$ & Sex & $n$ & $\begin{array}{c}\text { Age } \\
\text { (years) }\end{array}$ & BMI SDS & HbA1c (\%) & $\begin{array}{l}\text { Insulin dose } \\
\qquad(\mathrm{mU} / \mathrm{kg})\end{array}$ & $\begin{array}{c}\text { Duration of } \\
\text { diabetes (years) }\end{array}$ \\
\hline \multirow[t]{2}{*}{1} & $\mathrm{M}$ & 44 & $8.58 \pm 2.66$ & $0.07(-0.49 / 0.59)$ & $8.05 \pm 1.75$ & $0.63 \pm 0.23$ & $3.24 \pm 2.47$ \\
\hline & $\mathrm{F}$ & 26 & $9.65 \pm 3.03$ & $-0.17(-0.83 / 0.21)$ & $7.82 \pm 1.52$ & $0.80 \pm 0.28$ & $3.78 \pm 2.01$ \\
\hline \multirow[t]{2}{*}{2} & $M$ & 14 & $13.3 \pm 1.45$ & $-0.25(-0.65 / 0.70)$ & $9.46 \pm 4.46$ & $0.79 \pm 0.27$ & $7.53 \pm 4.08$ \\
\hline & $\mathrm{F}$ & 6 & $11.6 \pm 0.97$ & $0.13(-0.15 / 0.76)$ & $6.99 \pm 1.26$ & $0.86 \pm 0.15$ & $3.84 \pm 2.83$ \\
\hline \multirow[t]{2}{*}{3} & $M$ & 13 & $13.7 \pm 0.96$ & $0.15(0.04 / 0.43)$ & $8.79 \pm 2.17$ & $0.75 \pm 0.16$ & $3.89 \pm 2.50$ \\
\hline & $\mathrm{F}$ & 9 & $12.3 \pm 1.48$ & $-0.05(-0.44 / 0.26)$ & $9.69 \pm 3.30$ & $0.72 \pm 0.21$ & $5.93 \pm 3.91$ \\
\hline \multirow[t]{2}{*}{4} & $M$ & 17 & $15.0 \pm 1.47$ & $0.13(-0.17 / 1.0)$ & $9.18 \pm 1.85$ & $0.87 \pm 0.19$ & $7.18 \pm 3.61$ \\
\hline & $\mathrm{F}$ & 17 & $14.2 \pm 1.45$ & $0.29(-0.23 / 0.58)$ & $9.29 \pm 1.65$ & $0.75 \pm 0.29$ & $4.51 \pm 2.85$ \\
\hline \multirow[t]{2}{*}{5} & $\mathrm{M}$ & 26 & $16.7 \pm 1.22$ & $0.15(0.02 / 1.24)$ & $8.58 \pm 1.93$ & $0.74 \pm 0.20$ & $5.12 \pm 2.16$ \\
\hline & $\mathrm{F}$ & 38 & $15.8 \pm 1.55$ & $0.68(0.24 / 1.20)$ & $8.98 \pm 2.73$ & $0.76 \pm 0.26$ & $5.06 \pm 3.85$ \\
\hline
\end{tabular}

M, boys; F, girls. 


\section{Statistical analysis}

For statistical analysis, we used the STATISTICA 6.0 software program (StatSoft, Tulsa, USA). As data for BMI SDS, leptin, sOb-R and the sOB-R/leptin ratio were non-normally distributed, nonparametric statistical tests were used. Comparison of BMI SDS in individual Tanner stages was performed by analysis of variance as well as by the post-hoc Mann-Whitney U-test. Adjustment of parameters of the leptin axis for BMI was performed by analysis of covariance.

\section{Results}

\section{sOB-R and leptin serum concentrations in children with type $1 \mathrm{DM}$ during puberty}

A significant inverse relationship between sOB-R and Tanner stage was detectable in both genders ( $r=-0.30, P<0.01$ in boys; $r=-0.26, P=0.01$ in girls). The changes of sOB-R between the individual stages are shown in Table 2. However, serum concentrations of sOB-R did not reveal gender dependence in patients with diabetes as calculated by correlation analysis according to Spearman $(P=0.97)$. Furthermore, in the whole group of patients, we demonstrated statistically significant correlations $(P<0.02)$ between sOB-R and the duration of diabetes $(r=0.30)$, HbAlc levels $(r=0.32)$ and insulin dose $(r=0.18)$. In the subgroup of pubertal patients $(n=140$, Tanner stage of $>1$ ), these coefficients of correlation increased to $r=0.43$ (diabetes duration); $r=0.46$ (HbA1c level) (Fig. 1) and $r=0.28$ (insulin dose), with a statistical significance of $P<0.001$ in all comparisons. Multiple-regression analysis revealed that $\mathrm{HbA1c}(12.4 \%)$, height $(7.9 \%)$ and duration of diabetes $(8.7 \%)$ contributed to $29 \%$ variance of sOB-R in the diabetic children. Interestingly, leptin was not a significant predictor of sOBR. Leptin levels were significantly higher in girls with diabetes than in boys $(P<0.0001)$, with the exception of patients with Tanner stage 2 . In girls, serum leptin concentrations increased during pubertal development (Table 2). In boys, leptin levels increased only until Tanner stage 2 and decreased until the end of puberty (Table 2). Therefore, the relationship between leptin and Tanner stages was distinctly different between girls $(r=0.59 ; P<0.001)$ and boys $(r=-0.20, P<0.05)$. Consequently, compared with boys ( $r=0.28$; $P<0.01$ ), serum leptin levels demonstrated a stronger correlation with BMI SDS in girls $(r=0.60, P<0.001)$. In our cohort of patients, only HbA1C $(r=0.15, P=0.03)$ was significantly correlated to leptin levels, but not the duration of diabetes or the individual insulin dose. In the multipleregression analysis including age, BMI, height, Tanner stage, sOB-R and our diabetes-associated variables, only BMI, in boys, and BMI and Tanner stage, in girls, were significant independent predictors of the variation of leptin levels.

\section{Comparison of sOB-R and leptin between healthy children and children with diabetes}

To investigate the differences in sOB-R, leptin and the sOB-R/leptin ratio between healthy children and children with diabetes, we compared these parameters of our cohort with 526 healthy children aged 6-20 years (Table 2 and Figs 2-4). In almost all Tanner stages, sOB-R serum levels (Fig. 2) and sOB-R/leptin ratio data (Fig. 4) were significantly higher in children

Table 2 Data of the leptin axis (median, quartiles) from patients with type 1 diabetes mellitus (DM) by Tanner stage (TS) and compared to levels of healthy controls. Significant differences between both groups were demonstrated as ${ }^{\star} P<0.05$; ${ }^{\star \star} P<0.01$; ${ }^{\star \star \star} P<0.001$.

\begin{tabular}{|c|c|c|c|c|c|}
\hline TS (sex) & Group & $n$ & sOB-R (ng/ml) & Leptin (ng/ml) & sOB-R/leptin ratio \\
\hline 1 & DM & 44 & $59.3(50.3 / 76.8)$ & $3.00(2.18 / 3.59)^{\star}$ & $2.68(1.91 / 3.30)$ \\
\hline (boys) & Control & 58 & $57.6(44.4 / 69.3)$ & $1.96(1.31 / 3.56)$ & 3.39 (1.69/6.29) \\
\hline 1 & DM & 26 & $68.4(58.8 / 78.6)^{\star \star \star *}$ & $3.99(2.93 / 5.42)$ & $1.89(1.53 / 2.92)$ \\
\hline (girls) & Control & 53 & 53.7 (48.0/60.6) & $3.35(1.72 / 5.98)$ & $1.95(1.24 / 4.10)$ \\
\hline 2 & DM & 14 & $55.0(47.3 / 75.5)^{\star *}$ & $4.36(2.19 / 5.57)$ & $2.08(1.28 / 2.66)^{\star \star}$ \\
\hline (boys) & Control & 49 & $42.0(33.9 / 49.8)$ & $4.96(3.68 / 10.9)$ & $1.15(0.42 / 1.48)$ \\
\hline 2 & DM & 6 & $42.6(33.5 / 43.7)$ & $2.95(2.16 / 10.1)^{*}$ & $1.76(0.53 / 3.11)$ \\
\hline (girls) & Control & 49 & $43.2(36.6 / 50.1)$ & $6.84(4.81 / 11.9)$ & $0.66(0.42 / 1.21)$ \\
\hline 3 & DM & 13 & $48.2(38.4 / 61.5)^{\star \star}$ & $2.15(1.92 / 3.65)^{*}$ & $2.10(1.66 / 3.12)^{\star \star}$ \\
\hline (boys) & Control & 49 & $33.3(27.0 / 39.0)$ & $3.58(2.44 / 8.56)$ & $1.08(0.41 / 1.91)$ \\
\hline 3 & DM & 9 & $57.0(39.9 / 72.8)^{\star \star}$ & $3.68(3.19 / 8.66)$ & $1.11(0.68 / 2.87)^{\star}$ \\
\hline (girls) & Control & 50 & $34.1(29.1 / 37.8)$ & $6.77(4.89 / 9.40)$ & $0.55(0.40 / 0.87)$ \\
\hline 4 & DM & 17 & $55.1(44.2 / 74.2)^{\star \star \star}$ & $2.52(1.64 / 4.21)$ & $3.08(2.30 / 3.80)^{\star \star \star}$ \\
\hline (boys) & Control & 50 & $27.8(24.0 / 32.7)$ & $2.91(1.68 / 4.37)$ & $1.15(0.68 / 2.20)$ \\
\hline 4 & DM & 17 & $50.9(41.9 / 60.8)^{\star \star}$ & 7.33 (6.10/9.83) & $0.84(0.68 / 1.24)^{\star}$ \\
\hline (girls) & Control & 50 & $37.8(30.0 / 41.7)$ & $6.82(5.60 / 11.3)$ & $0.58(0.43 / 0.88)$ \\
\hline 5 & DM & 26 & $40.0(34.2 / 54.7)^{\star \star \star}$ & 2.05 (1.58/3.27) & $2.51(1.49 / 4.42)^{\star \star \star}$ \\
\hline (boys) & Control & 56 & $29.1(23.0 / 38.0)$ & $1.87(1.21 / 2.77)$ & $2.07(1.17 / 3.19)$ \\
\hline 5 & DM & 38 & $53.0(34.6 / 61.9)^{\star \star \star}$ & $13.1(8.45 / 17.3)$ & $0.50(0.29 / 0.93)^{*}$ \\
\hline (girls) & Control & 62 & $29.4(24.3 / 38.7)$ & $9.04(6.50 / 14.8)$ & $0.36(0.22 / 0.56)$ \\
\hline
\end{tabular}




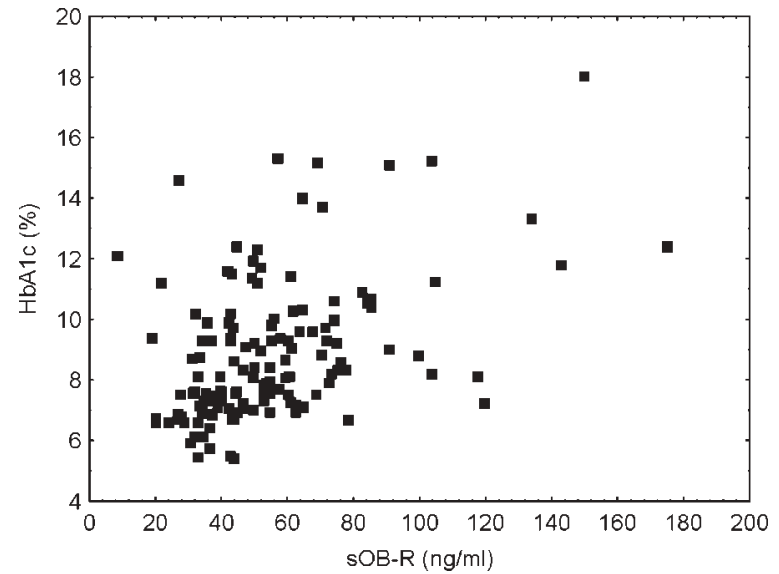

Figure 1 Comparison between levels of sOB-R in serum and $\mathrm{HbA1c}$ in blood of pubertal patients with type 1 diabetes mellitus ( $n=140$, Tanner stage of $>1)$. The coefficient of correlation according to Spearman was calculated as $r=0.46(P<0.001)$.

with diabetes than in healthy children $(P<0.05)$. In contrast to the group of healthy children, which demonstrated decreasing sOB-R levels and ratio data through puberty, sOB-R levels remained high in children with diabetes throughout this period. The
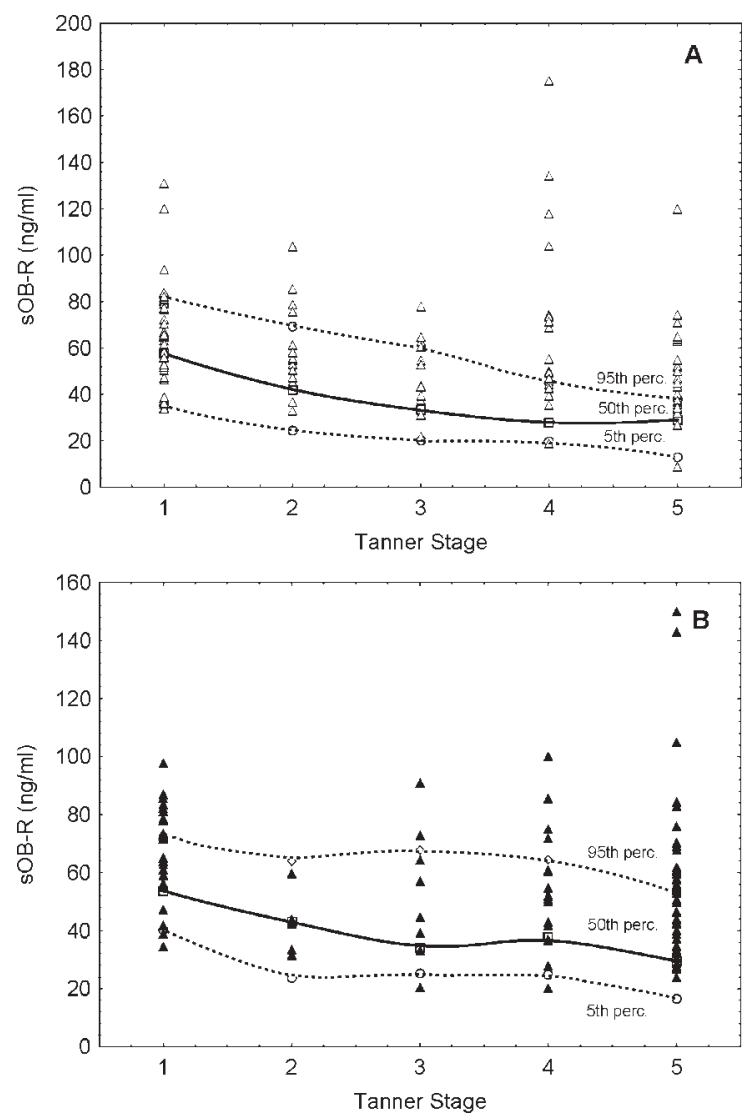

Figure 2 sOB-R levels of male (A) and female (B) children and adolescents with type 1 diabetes mellitus compared with the 5th (dotted line), 50th (solid line) and 95th (dotted line) percentiles of healthy children by pubertal stage according to Tanner stage. distinctly increased values of the sOB-R/leptin ratio of children with diabetes was predominantly due to their already mentioned high sOB-R levels, but also to their approximately unchanged leptin levels in comparison with healthy children. Only in Tanner stage 1 did boys with diabetes (Fig. 3A) have significantly higher leptin levels than our controls $(P<0.05)$. In contrast, leptin levels of boys with Tanner stage 3 were significantly lower in the diabetic patients than in healthy subjects $(P=0.015)$. Female type $1 \mathrm{DM}$ patients (Fig. 3B) demonstrated lower leptin values in serum of Tanner stage $2(P=0.02)$. All these differences remained significant if leptin levels were adjusted for the BMI values of our subjects.

\section{Discussion}

In this study, we show for the first time that important parameters characterizing the metabolic state of type 1 $\mathrm{DM}$, like HbA1c levels, insulin dosage and the duration of diabetes, are correlated with the serum sOB-R level. Therefore, children with poor glycemic control reveal a massive increase in the sOB-R/leptin ratio. The latter parameter reflects the molar excess of sOB-R
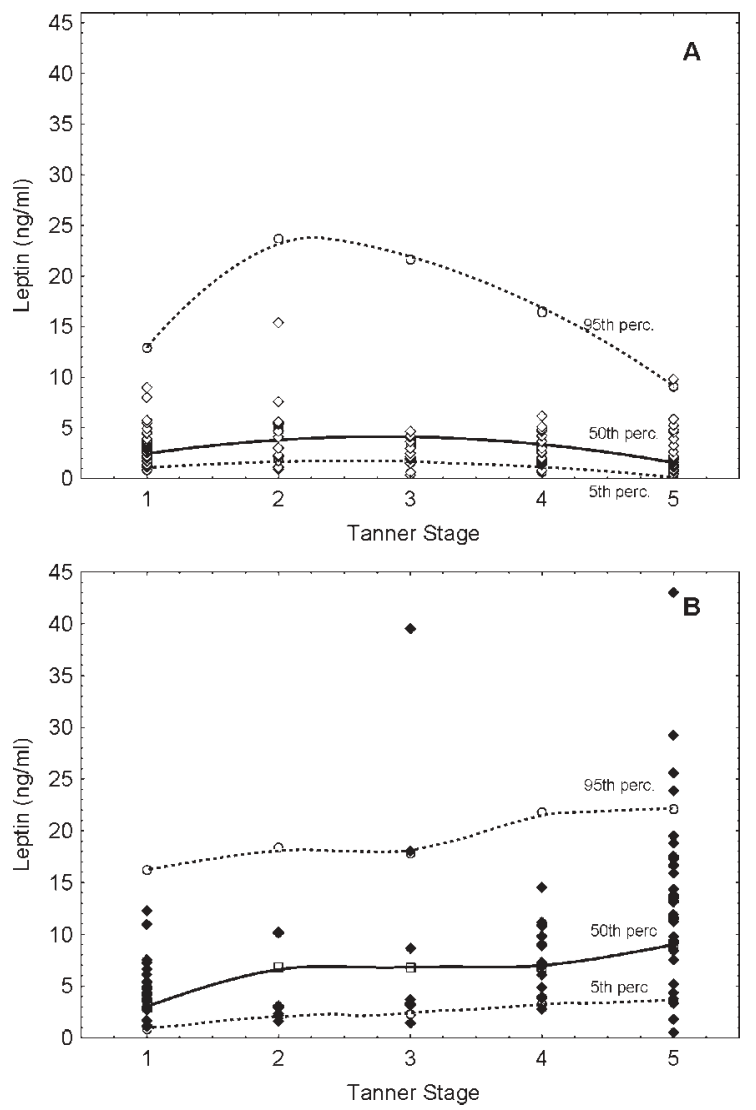

Figure 3 Leptin levels of male (A) and female (B) children and adolescents with type 1 diabetes mellitus compared with the 5th (dotted line), 50th (solid line) and 95th (dotted line) percentiles of healthy children by pubertal stage according to Tanner stage. 

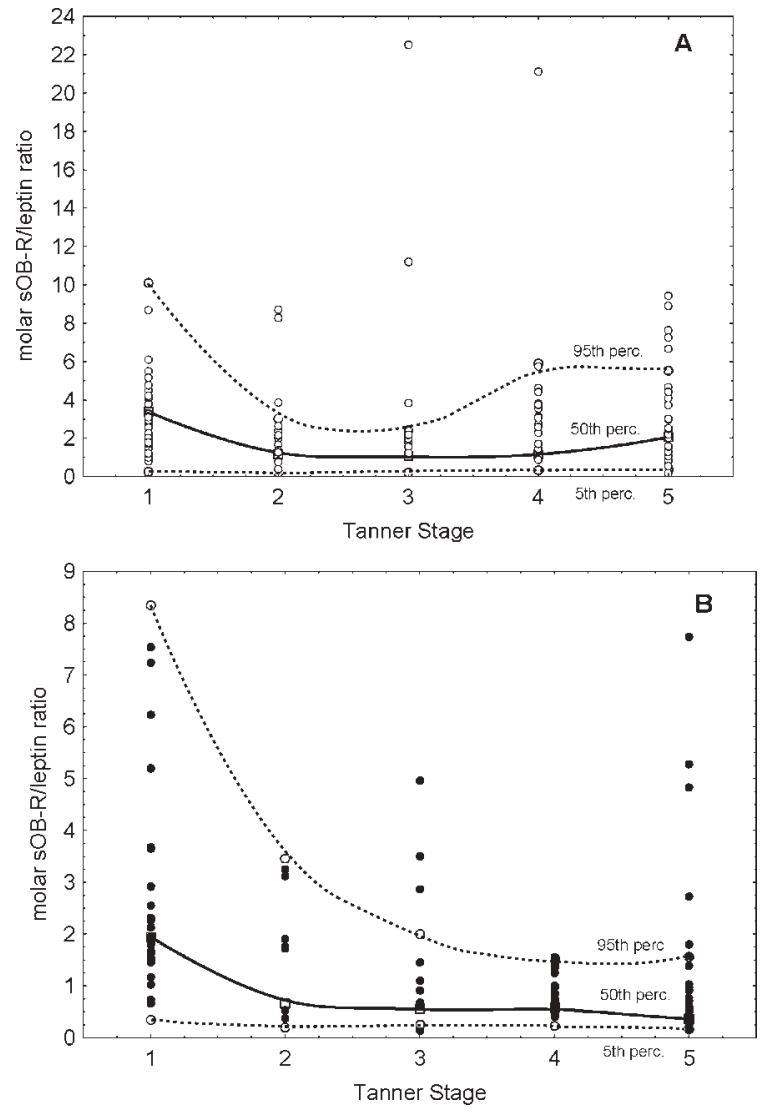

Figure 4 Data of the ratio between sOB-R and leptin levels of male $(A)$ and female $(B)$ children and adolescents with type 1 diabetes mellitus compared with the 5th (dotted line), 50th (solid line) and 95th (dotted line) percentiles of healthy children by pubertal stage according to Tanner stage.

over leptin in blood, a measure of the leptin action as validated by in vitro cell experiments (23). A high level of this ratio may demonstrate reduced leptin bioactivity. Whereas the elevation of sOB-R levels as well as of sOB-R/leptin ratios in diabetes is a new finding of our study, the levels of leptin and the relationships between leptin, BMI and Tanner stage are consistent with the findings of other authors. Thus, the gender-specific differences in the leptin course during puberty can partially be explained by the differences in body composition: girls have a higher percentage of fat mass and boys gain more muscle mass. The latter appears to be caused by increased androgen serum levels, which may by themselves inhibit peripheral leptin secretion $(6,7,11,30,31)$. Furthermore, the leptin concentrations in our study were mainly comparable to the results of healthy controls, except boys in subgroups with Tanner stages 1 and 3 and girls in the subgroup with Tanner stage 2 . We speculate that these differences were due to the relatively low number of female subjects in Tanner stage 2 or were related to the degree of individual insulin treatment: insulinopenia, a lowered insulin sensitivity or compliance problems may lead to decreased levels of leptin
$(18,21)$. On the other hand, hyperinsulinemia caused by exogenous insulin treatment, concurring with a marginal remaining insulin secretion, especially during the first year after diagnosis of type 1 DM, could be associated with an increase of leptin concentrations $(15,18,22)$.

In contrast to sOB-R, no important parameters characterizing the metabolic state of type 1 DM, such as $\mathrm{HbA1c}$ levels, insulin dosage and the duration of diabetes, were significant independent predictors for serum leptin levels in the multivariate calculations. Therefore, the metabolic situation may have had only a minor influence on the leptin values of these patients. The finding that the leptin concentrations of our cohort were not essentially different from the control data supports this hypothesis. However, as observed for our healthy subjects (data not shown), BMI and Tanner stage are the major independent predictors also in the diabetic state. The distinctly elevated levels of sOB-R and of the SOB-R/leptin ratio in insulin-treated subjects raised the question of whether sOB-R inhibits leptin bioactivity, leading to leptin resistance and possibly weight gain. Although we cannot definitely answer this question, the direct correlation of sOB-R (but not of leptin) with insulin dose, HbAlc and the duration of diabetes, as measured in our study, suggests that metabolic decompensation may have an indirect (mediated by sOB-R) impact on leptin action in type 1 DM. Leptin, the potential regulator for sOB-R shedding in healthy subjects $(32,33)$, seems to have no relevant effect on sOB-R secretion in diabetes. A possible explanation for the increase of sOB-R observed in our patients may be the well-known induction of protein kinase $C$ (PKC) activity in the diabetic state. PKC can induce A disintegrin and metalloprotease (ADAM) 17 (34), which is able to shed the extracellular domain of cytokine-like hormone receptors, such as growth hormone-receptor and the OB-R, leading to increased levels of the soluble receptor in our patients. Interestingly, Lewandowski et al. (35) have found an increased level of sOB-R in pregnant women with diabetes compared with pregnant nondiabetic women. This observation supports our findings. Otherwise, Diamond et al. (36) investigated the leptin-binding activity of teenagers with diabetes ( 8 girls, 10 boys) without detecting any differences from healthy children. The contradiction between the results of this study and our findings may be explained by the low number of subjects, the lack of consideration of pubertal stage and the use of size-exclusion chromatography to quantify leptin binding, which appears to be a relatively unspecific analytic method for the quantification of sOB-R. The biologic consequences of increased sOB-R levels in diabetes are unclear so far. Due to its binding affinity, which is comparable to the binding affinity of the membrane leptin receptor $(23,37)$, sOB-R may modulate leptin action in two ways. First, sOB-R could inhibit the binding of leptin to specific membrane 
receptors by its direct competition for the ligand. In a recent paper, our group was able to show that a 10fold molar excess of sOB-R over leptin distinctly suppressed leptin bioactivity in vitro (23). An up to 20fold molar excess has been shown by this study. Thus, we hypothesize that this molar excess of sOB-R contributes to leptin insensitivity, which may support weight gain and a decrease of energy consumption in a catabolic condition such as newly diagnosed DM. The second mechanism would involve complexes of leptin and sOB-R inhibiting the renal elimination of leptin, leading to an increased bioavailability of this hormone. However, the latter mechanism would conflict with the increased weight gain of diabetic children during puberty. In conclusion, the finding of elevated sOB-R levels in children and adolescents with diabetes extends to the understanding of the molecular basis for putative derangement of body weight control in diabetes at a young age. To clarify the mechanism of sOB-R secretion and to assess the detailed pathophysiological effect of the molar excess between sOB-R and leptin in diabetes, more detailed investigations should be made. Most interestingly, the question of whether or not patients with leptin insensitivity have a better chance to overcome a state of metabolic decompensation in DM should be a goal for further clinical studies.

\section{Acknowledgements}

The authors are very grateful to Mrs Jaeschke and Mrs Liebig for the excellent technical assistance. This work was supported by the Bundesministerium für Bildung und Forschung $(\mathrm{BMB}+\mathrm{F})$, Interdisciplinary Centre for Clinical Research (IZKF) at the University of Leipzig (Project B15), Leipzig, Germany.

\section{References}

1 Zhang Y, Proenca R, Maffei M, Barone M, Leopold L \& Friedmann JM. Positional cloning of the mouse obese gene and its human homologue. Nature 1994372 425-432.

2 Pelleymounter MA, Cullen MJ, Baker MB, Hecht R, Winters D, Boone T \& Collins F. Effects of obese gene product on body weight regulation in ob/ob mice. Science $1995 \mathbf{2 6 9} 540-543$.

3 Halaas JL, Gajiwala KS, Maffei M, Cohen SL, Chait BT, Rabinowitz D, Lallone RL, Burley SK \& Friedman JM. Weight reducing effects of the plasma protein encoded by the obese gene. Science 1995 $269543-546$.

4 Campfield LA, Smith FJ, Guisez Y, Devos R \& Burn P. Recombinant mouse OB protein: evidence for a peripheral signal linking adiposity and central neural networks. Science $1995 \mathbf{2 6 9}$ 546-549.

5 Stephens TW, Basinski M, Bristow PK, Bue-Valleskey JM, Burgett SG, Craft L, Hale J, Hoffman J, Hsiung HM, Kriauciunas A, MacKellar W, Rosteck PR, Schoner B, Smith D, Tinsley FC, Zhang XY \& Heimann M. The role of neuropeptide $\mathrm{Y}$ in the antiobesity action of the obese gene product. Nature $1995377530-532$.

6 Clayton PE, Gill MS, Hall CM, Tillmann V, Whatmore A \& Price DA. Serum leptin through childhood and adolescence. Clinical Endocrinology 199746 727-733.
7 Friedman JM \& Halaas JL. Leptin and the regulation of body weight in mammals. Nature 1998395 763-770.

8 Kiess W, Reich A, Meyer K, Glasow A, Deutscher J, Klammt J, Yang Y, Müller G \& Kratzsch J. A role for leptin in sexual maturation and puberty. Hormone Research 199951 55-63.

9 Ricardo V, Garcia-Mayor RV, Andrade MA, Rios M, Lage M, Dieguez C \& Casanueva F. Serum leptin levels in normal children: relationship to age, gender, body mass index, pituitary-gonadal hormones, and pubertal stage. Journal of Clinical Endocrinology and Metabolism $1997 \mathbf{8 2} 2849-2855$.

10 Jockenhövel F, Blum WF, Vogel E, Englaro P, Müller-Wieland D, Reiwein D, Rascher W \& Krone W. Testosterone substitution normalizes elevated serum leptin levels in hypogonadal men. Journal of Clinical Endocrinology and Metabolism $1997 \mathbf{8 2}$ $2510-2513$.

11 Blum WF, Englaro P, Hanitsch S, Juul A, Hertel NT, Müller J, Skakkebaek NE, Heiman ML, Birkett M, Attanasio AM, Kiess W \& Rascher W. Plasma leptin levels in healthy children and adolescents: dependence on body mass index, body fat mass, gender, pubertal stage, and testosterone. Journal of Clinical Endocrinology and Metabolism $1997 \mathbf{8 2} 2904-2910$.

12 Adan L, Bussières L, Trivin C, Souberbielle JC \& Brauner R. Effect of short-term testosterone treatment on leptin concentration in boys with pubertal delay. Hormone Research 199952 109-112.

13 Thon A, Heinze E, Feilen KD, Holl RW, Schmidt H, Koletzko S, Wendel U \& Nothjunge J. Development of height and weight in children with diabetes mellitus: report on two prospective multicentre studies, one cross-sectional, one longitudinal. European Journal of Pediatrics $1992151258-262$.

14 Brown M, Ahmed ML, Clayton KL \& Dunger DB. Growth during childhood and final height in type 1 diabetes. Diabetic Medicine $199411182-187$.

15 Luna R, Garcia-Mayor RV, Lage M, Andrade MA, Barreiro J, Pmobo M, Dieguez C \& Casanueva FF. High serum leptin levels in children with type 1 diabetes mellitus: contribution of age, BMI, pubertal development and metabolic status. Clinical Endocrinology $1999 \mathbf{5 1} 603-610$.

16 Dunger DB, Ahmed ML \& Ong KKL. Growth and body composition in type 1 diabetes mellitus. Hormone Research $2002 \mathbf{5 8}$ $66-71$.

17 Ahmed ML, Ong KKL, Watts AP, Morrell DL, Preece MA \& Dunger DB. Elevated leptin levels are associated with excess gains in fat mass in girls, but not boys, with type 1 diabetes mellitus: longitudinal study during adolescence. Journal of Clinical Endocrinology and Metabolism 2001 86 1188-1193.

18 Kiess W, Anil M, Blum WF, Englaro P, Juul A, Attanasio A, Dötsch J \& Rascher W. Serum leptin levels in children and adolescents with insulin-dependent diabetes mellitus in relation to metabolic control and body mass index. European Journal of Endocrinology $1998138501-509$.

19 Verotti A, Basciani F, Morgese G \& Chiarelli F. Leptin levels in nonobese and obese children and young adults with type 1 diabetes mellitus. European Journal of Endocrinology $199813949-53$.

20 Flück CE, Kuhlmann BV \& Mullis PE. Insulin increases serum leptin concentrations in children and adolescents with newly diagnosed type 1 diabetes mellitus with and without ketoacidosis. Diabetologia 199942 1067-1070.

21 Kirel B, Dogruel N, Korkmaz Ü, Sultan Kilic F, Özdamar K \& Ucar B. Serum leptin levels in type 1 diabetic and obese children: relation to insulin levels. Clinical Biochemistry 200033 475-480.

22 Soliman AT, Omar M, Assem HM, Nasr IS, Rizk MM, El Matary W \& El Alaily RK. Serum leptin concentrations in children with type 1 diabetes mellitus: relationship to body mass index, insulin dose, and glycemic control. Metabolism 200251 292-296.

23 Lammert A, Kiess W, Böttner A, Glasow A \& Kratzsch J. Soluble leptin receptor represents the main leptin binding activity in human blood. Biochemical and Biophysical Research Communications $2001 \mathbf{2 8 3} 982-988$. 
24 Kratzsch J, Lammert A, Böttner A, Seidel B, Mueller G, Thiery J, Hebebrand W \& Kiess W. Circulating soluble leptin receptor and free leptin index during childhood, puberty, and adolescence. Journal of Clinical Endocrinology and Metabolism 200287 4587-4594.

25 Zastrow O, Seidel B, Kiess W, Thiery J, Keller E, Böttner A \& Kratzsch J. The soluble leptin receptor is crucial for leptin action: evidence from clinical and experimental data. International Journal of Obesity $2003271472-1478$.

26 American Diabetes Association. Clinical Practice Recommendations 2004. Diabetes Care 2004 (Suppl 1).

27 Kromeyer-Hauschild K, Wabitsch M, Kunze D, Geller F, Gei HC, Hesse V, von Hippel A, Jaeger U, Johnsen D, Korte W, Menner K, Müller G, Müller JM, Niemann-Pilatus A, Remer T, Schaefer F, Wittchen HU, Zabransky S, Zellner K, Ziegler A \& Hebebrand J. Perzentilen für den Body-Mass-Index für das Kindes-und Jugendalter unter Heranziehung verschiedener deutscher Stichproben. Monatsschrift Kinderheilkunde $2001149807-818$.

28 Tanner JM. Growth at Adolescence, 2nd edn. Oxford: Blackwell Scientific, 1962.

29 Kratzsch J, Berthold A, Lammert A, Reuter W, Keller E \& Kiess W. A rapid, quantitative immunofunctional assay for measuring human leptin. Hormone Research 200257 127-132.

30 Garcia-Mayor RV, Andrade MA, Rios M, Lage M, Dieguez C \& Casanueva FF. Serum leptin levels in normal children: relationship to age, gender, body mass index, pituitary-gonadal hormones, and pubertal stage. Journal of Clinical Endocrinology and Metabolism 199782 2849-2855.

31 Ankarberg-Lindgren C, Dahlgren J, Carlsson B, Rosberg S, Carlsson L, Wikland KA \& Norjavaara E. Leptin levels show diurnal variation throughout puberty in healthy children, and follow a gender-specific pattern. European Journal of Endocrinology $200114543-51$.
32 Mann DR, Johnson AO, Gimpel T \& Castracane VD. Changes in circulating leptin, leptin receptor, and gonadal hormones from infancy until advanced age in humans. Journal of Clinical Endocrinology and Metabolism $2003 \mathbf{8 8} 3339-3345$.

33 Chan JL, Bluher S, Yiannakouris N, Suchard MA, Kratzsch J \& Mantzoros CS. Regulation of circulating soluble leptin receptor levels by gender, adiposity, sex steroids, and leptin: observational and interventional studies in humans. Diabetes $2002 \mathbf{5 1}$ 2105-2112.

34 Zhang Y, Jiang J, Black RA, Baumann G \& Frank SJ. Tumor necrosis factor-alpha converting enzyme (TACE) is a growth hormone binding protein (GHBP) sheddase: the metalloprotease TACE/ADAM-17 is critical for (PMA-induced) GH receptor proteolysis and GHBP generation. Endocrinology $20001414342-4348$.

35 Lewandowski K. Horn R, O'Callaghan CJ, Dunlop D, Medley GF, O'Hare P \& Brabant G. Free leptin, bound leptin, and soluble leptin receptor in normal and diabetic pregnancies. Journal of Clinical Endocrinology and Metabolism $1998 \mathbf{8 4} 300-306$.

36 Diamond F, Eichler D, Mayes D, Jorgensen V, Duckett G, Hu C, Cuthbertson D \& Root A. Leptin binding activity in plasma of nondiabetic and diabetic adolescents and hormonal data. Journal of Pediatric Endocrinology and Metabolism 200013 141-148.

37 Liu C, Liu XJ, Barry G, Ling N, Maki R \& DeSouza EB. Expression and characterization of a putative high affinity human soluble receptor. Endocrinology $19971383548-3554$.

Received 27 April 2004

Accepted 12 July 2004 